\title{
Model predictive Control of a Double Stage AC-DC Converter for Grid-Interface of Vanadium Flow Batteries
}

\author{
Salvatore Riccardo Di Salvo \\ Electrical, Computer and Biomedical Engineering Dept., \\ University of Pavia \\ Pavia, Italy \\ salvatorericca.disalvo01@universitadipavia.it \\ Jacopo Riccio \\ Faculty of Eng. Electrical and Electronics Engineering, \\ The University of Nottingham \\ Nottingham, UK \\ jacopo.riccio1@nottingham.ac.uk \\ Pericle Zanchetta \\ Electrical, Computer and Biomedical Engineering Dept., \\ University of Pavia \\ Pavia, Italy \\ pericle.zanchetta@unipv.it
}

\begin{abstract}
This paper presents a multi-objective model predictive control algorithm to implement the control of a bidirectional double stage ac-dc power conversion system, used to interface a Vanadium redox flow battery with a power grid. The converter topology is based on two stages conversion, consisting in a dual active bridge, controlled using the phase shift modulation, in cascade with a grid-connected inverter/rectifier. In order to control the charge and discharge of the battery, the dc-link and the grid current, a multi-objective predictive control is introduced embedding the control of both conversion stages in a single control action. The computed steady state and dynamic performance are compared to what can be achieved with standard controllers. The proposed algorithm is tested in simulation using a model developed with the PLECS block library on Matlab/Simulink.

Index Terms-dual active bridge, energy storage system, model predictive control, three phase inverter/rectifier, vanadium redox flow battery
\end{abstract}

\section{INTRODUCTION}

In the last decades, the research interest in microgrid has been steadily growing due to several advantages they bring to modern power systems, such as system efficiency and reliability improvements, increased power quality and continuity of service [1]. Power electronics is an enabling technology for the development of microgrids because it allows the reliable integration of renewable energy sources (e.g. photovoltaic, wind turbines etc.) and various types of energy storage devices (ESDs). ESDs, which are considered a key component for modern microgrids, especially in the case of electrical systems characterized by weak inertia, where the

\author{
Matteo Bulzi \\ Electrical, Computer and Biomedical Engineering Dept., \\ University of Pavia \\ Pavia, Italy \\ matteo.bulzi01@universitadipavia.it
}

\author{
Riccardo Leuzzi \\ Electrical, Computer and Biomedical Engineering Dept., \\ University of Pavia \\ Pavia, Italy \\ riccardo.leuzzi@unipv.it \\ Norma Anglani \\ Electrical, Computer and Biomedical Engineering Dept., \\ University of Pavia \\ Pavia, Italy \\ nanglani@unipv.it
}

intrinsic short-time power variability of distributed renewable energy sources may lead to voltage and frequency oscillations that would degrade the power quality and eventually cause system instability [2]. In this framework, Vanadium Redox Flow Batteries (VRFBs) are particularly promising for a wide range of microgrids applications thanks to many desired features such as long life (over 20 years), very high number of charge/discharge cycles (over 20,000), independent scalability of power and energy capacity and low operating temperature (ambient temperature). In addition, VRFBs have long discharge time, high round trip efficiency $(75 \%)$, high short-time overloading, and response time in the order of milliseconds, which are crucial characteristics when dealing with renewable energy sources [3]. Finally, compared to other technologies, the maintenance cost and the initial capital investment are lower (expenditure capital less than $663 €_{2016} / \mathrm{kWh}$ ) [4].

Usually, ESDs require a bidirectional connection with the microgrid to guarantee the charging and discharging cycles. A well-known architecture for interfacing ESDs with the power grid is composed of a bidirectional three-phase grid-side converter (GSC) and a dual active bridge (DAB) in a doublestage configuration, as shown in Fig. 1. This ac-dc converter architecture represents a good solution because its two-stage structure (i.e., ac-dc $+\mathrm{dc}-\mathrm{dc}$ converter) also allows the direct connection of renewables (e.g., photovoltaics) directly on the available high-voltage dc-bus, eventually removing additional conversion stages. This configuration was presented for the first time in [5] to overcome the traditional configuration for ESDs integration based on a line-frequency transformer, a three-phase GSC and a bidirectional chopper. Thanks to the use of the DAB, this topology features a high-frequency transformer within the dc-dc stage, providing galvanic isolation and avoiding the use of bulky $50 \mathrm{~Hz}$ transformers. Moreover, 


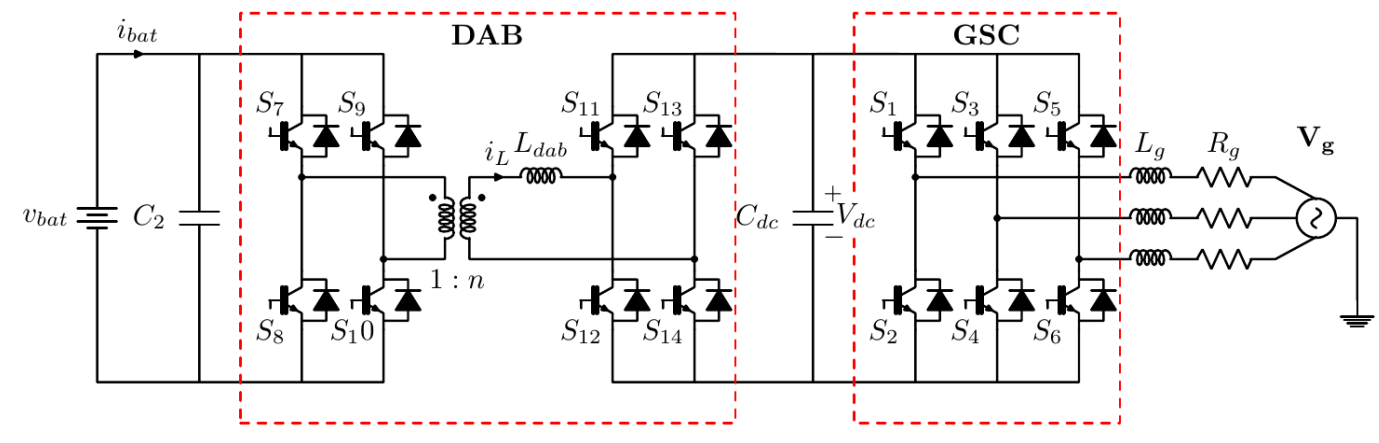

Fig. 1: Schematic of the double stage ac-dc power converter.

the DAB can be controlled to achieve soft-switching operation, this condition allows to drastically reduce switching losses, enabling higher switching frequency operations and consequently reduce the size of filters and other passive components of the converter [6].

According to the classification of grid-tied converters given in [7], the topology presented in this paper can be defined as a grid-feeding converter. Conventionally grid-feeding GSC uses PI controllers for voltage oriented control (VOC) [7]. The VOC technique exploits two cascaded controllers, the outer control loop regulates the dc-link voltage, while the inner loop controls the grid current. VOC is usually applied in the $d q 0$ reference system, so it needs Phase-Locked Loop (PLL) for grid synchronization the converter's output with the grid. PI controllers are also employed to control the bidirectional power transfer across the DAB [8]. In particular, the ESD current is controlled to ensure safe charge and discharge of the battery.

In the last three decades, due to the growing availability of faster microprocessors with higher computational capabilities, new and more advanced control techniques have been proposed [9]. Model Predictive Control (MPC) has proven to be among the most promising approaches in the field of power electronics and drives $[10,11]$ mainly due to two reasons: the existence of good mathematical models to predict the behaviour of power conversion system [12] and its fast dynamic response. In particular, the MPC approach can be applied to a wide variety of systems, including multi-inputmulti-output; it can deal with non-linearities and include hard and soft constraints and modulator dead-times into the control law. Moreover, it can merge cascaded controllers in a single control action achieving a faster dynamic response than conventional controllers [13].

In the literature there are several applications of the MPC strategy for three-phase converters. Originally, MPC was applied to the control of electrical drives as in [14], where a predictive current control strategy for a three-phase inverter feeding a permanent magnet synchronous motor is presented. In [15] a predictive power control scheme controls an active front end rectifier. A finite control set approach model predictive control (FCS-MPC) for a grid-connected three-phase inverter was presented in [16]. FCS-MPC established as an important branch of the predictive controllers family thanks to its low design complexity (prediction horizon of 1 step) and the advantage of direct application of the control action to the converter, without the modulation stage [17].

On the contrary, the control of the DAB through MPC algorithms has not been thoroughly investigated yet, even though it appears a promising trend. Recently, Chen et Al. focused on an MPC battery current controller for a DAB interfacing a ESD into a naval dc microgrid [18]. A multiobjective MPC was proposed in [19] to control the battery current and the dc-link voltage of a dc microgrid, using a moving discretized control set MPC (MDCS-MPC) to control the phase shift of the DAB.

The purpose of this work is to design a MPC algorithm to control the afore-mentioned converter topology with a single optimal control action at each time step, based on the principles of both the FCS-MPC and the MDCS-MPC. The aim is to regulate the dc-link voltage between the GSC, the battery current during charge and discharge, and the active and reactive power exchanged with the microgrid. A comparison between the performance achieved by the proposed MPC and conventional PI-based schemes is then carried out.

This paper is structured as follows: section II presents the MPC algorithm, section III shows and discuss the static and dynamic performance of the proposed MPC controller, finally section IV draws the conclusion of the work.

\section{DESCRIPTION OF THE PROPOSED MPC ALGORITHM}

The model's equation considered for the chosen converter topology, Fig. 1, derives from [5]. The block diagram of the proposed MPC control system is shown in Fig. 2. The input references for the controller are the desired dc-link voltage $v_{d c}^{*}(k)$, battery current $i_{b a t}^{*}(k)$ and reactive power $Q_{a c}^{*}(k)$. The outputs of the controller are the optimal gate signals for the GSC and the optimal phase shift for the DAB. The former are directly applied to the GSC, since the FCS-MPC approach

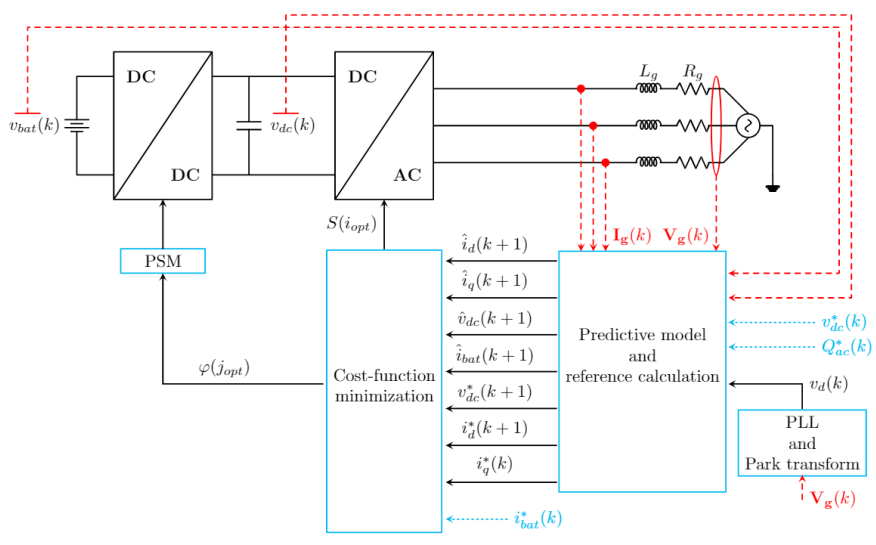

Fig. 2: Block diagram of the MPC system. 
eliminates the need for a modulation stage, while the latter is processed by the phase-shift modulator (PSM).

In order to control the active and reactive power exchanged with the microgrid, electrical quantities are represented into the synchronous reference frame $d q 0$, hence a PLL is necessary to perform the park transformation. In particular, the $d$-axis current component controls the active power while the $q$-axis current controls the reactive power. The cost function of the MPC is formulated considering the sum of the squared errors between the references and predictions of the controlled variables and it is calculated as

$$
\begin{aligned}
g & =\left[\frac{v_{d c}^{*}(k+1)-\hat{v}_{d c}(k+1)}{v_{d c}^{*}(k+1)}\right]^{2}+\left[\frac{i_{d}^{*}(k+1)-\hat{i}_{d}(k+1)}{i_{d}^{*}(k+1)}\right]^{2} \\
& +\left[\frac{i_{q}^{*}(k)-\hat{i}_{q}(k+1)}{i_{q}^{*}(k)}\right]^{2}+\left[\frac{i_{b a t}^{*}(k)-\hat{i}_{b a t}(k+1)}{i_{b a t}^{*}(k)}\right]^{2}
\end{aligned}
$$

where $v_{d c}^{*}(k+1)$ and $i_{d}^{*}(k+1)$ are the dc-link voltage and the $d$-axis current one-step-ahead references, at the instant $k+1, i_{q}^{*}(k)$ and $i_{\text {bat }}^{*}(k)$ are the $q$-axis and battery currents references at $k$-th instant. While $\hat{i}_{d}(k+1), \hat{i}_{q}(k+1), \hat{v}_{d c}(k+1)$ and $\hat{i}_{b a t}(k+1)$ are the predictions calculated by the model at the time step $k+1$. Because of the existence of constrains related to the dc-link voltage and active power control dynamics, in (1) proper values calculated at the instant $k+1$ are considered for the references associated to the dc-link voltage and to the $d$-axis current. These constrains do not hold for the reactive power and the battery current control, therefore the corresponding reference values at the instant $k$ are used.

To compensate for the different nature of the controlled variables, each error term has been normalized by the corresponding reference value such that all terms in (1) have comparable magnitude. The flowchart, given in Fig. 3, summarises the working principle of the adopted control algorithm, whose parts are described in detail in the following.

\section{A. Measurements and References calculation}

At time instant $k$, the controller acquires the measured values of the three-phase grid voltage $\mathbf{V}_{g}(k)$, grid current $\mathbf{I}_{g}(k)$, dc-link voltage $v_{d c}(k)$ and battery voltage $v_{b a t}(k)$, and it calculates the references for the $d$ - and $q$-axis current components, $i_{d}^{*}(k+1)$ and $i_{q}^{*}(k)$ respectively, and for the dc-link voltage $v_{d c}^{*}(k+1)$.

The voltage level across the dc-link capacitor of the GSC is directly coupled to the active power exchanged with the grid. Therefore, to achieve a robust and stable operation of the system it is important to ensure that the voltage control loop has a sufficiently slower dynamics than the power loop. In order to slow down the dynamics of the voltage control, when calculating $v_{d c}^{*}(k+1)$, a parameter $N$ is introduced to filter the signal before it is applied to the controller. The new reference is calculated as

$$
v_{d c}^{*}(k+1)=v_{d c}(k)+\frac{1}{N}\left[v_{d c}^{*}(k)-v_{d c}(k)\right]
$$

therefore the reference value considered at instant $k+1$ for the dc-link voltage is obtained by allowing the converter to reach the desired value $v_{d c}^{*}$ linearly in $N$ steps. The parameter $N$ is an important design parameter [20] that requires proper tuning. The design of $\mathrm{N}$ is discussed in Section II-C.
The power balance at the dc-link shown in Fig.4 is used to calculate the reference for the active power $P_{a c}^{*}(k+1)$ at the instant $k+1$ as

$$
P_{a c}^{*}(k+1)=P_{b a t}^{*}(k)+P_{d c}^{*}(k+1)
$$

where $P_{\text {bat }}^{*}$ is the power delivered/absorbed by the ESD and $P_{d c}^{*}$ is the power required by the dc-link.

Knowing the desired battery current $i_{b a t}^{*}(k)$, the battery power reference $P_{b a t}^{*}(k)$ can be calculated as in (4). It is worth mentioning that the $N$ parameter does not affect the dynamics of the battery current control and the calculation of the references for the battery current and power.

$$
P_{b a t}^{*}(k)=v_{b a t}(k) i_{b a t}^{*}(k)
$$

The dc-link power reference is given by

$$
P_{d c}^{*}(k+1)=v_{d c}^{*}(k+1) i_{d c}^{*}(k+1)
$$

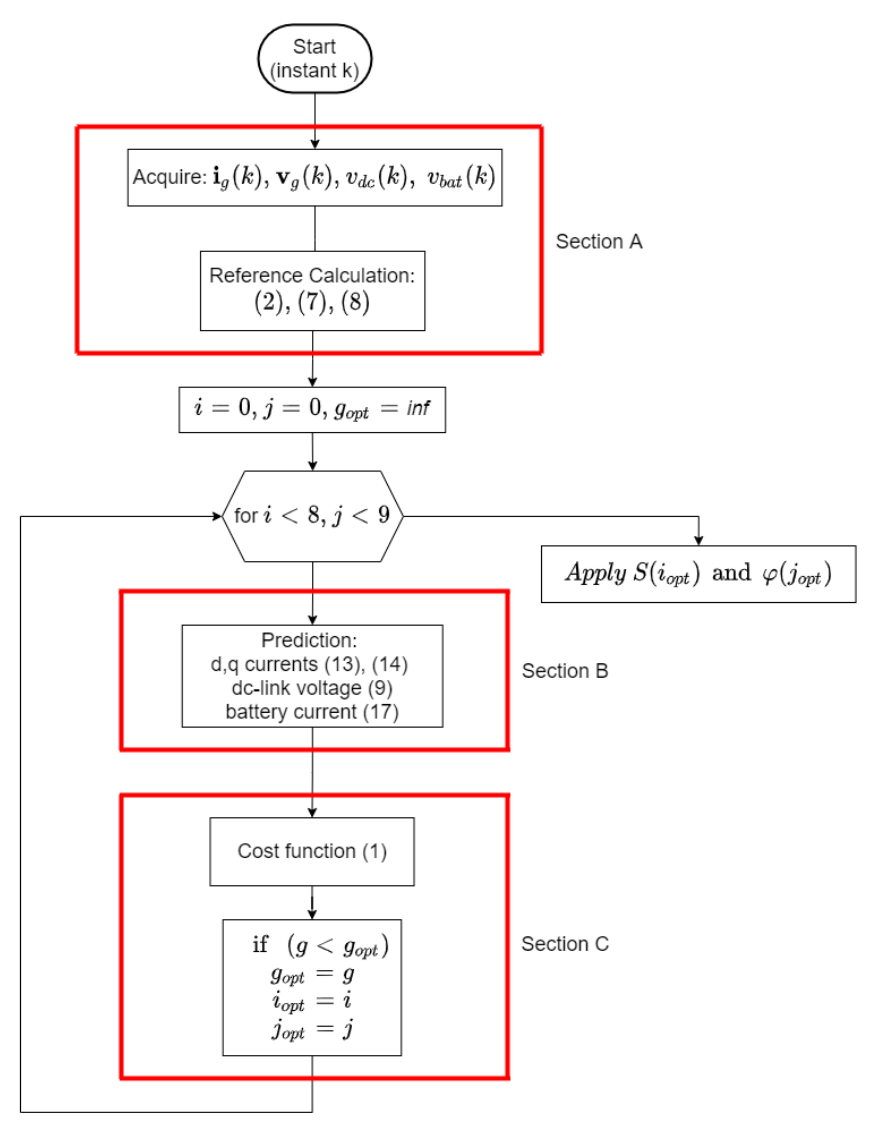

Fig. 3: Flowchart of the MPC algorithm.

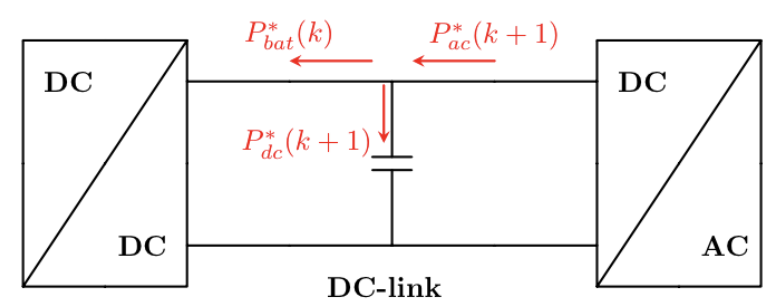

Fig. 4: Power balancing at the dc-link: the case where the power is absorbed from the grid and flows toward the battery is depicted. 
where the dc-link current reference $i_{d c}^{*}(k+1)$, similarly to what was done in [20], can be calculated as

$$
i_{d c}^{*}(k+1)=\frac{C_{d c}}{T_{s}}\left[v_{d c}^{*}(k+1)-v_{d c}(k)\right] .
$$

The $d$-axis current one-step-ahead reference and $q$-axis current reference $i_{q}^{*}(k)$ are calculated from the reference active and reactive power, $P_{a c}^{*}(k+1)$ and $Q_{a c}^{*}(k)$, as

$$
\begin{gathered}
i_{d}^{*}(k+1)=\frac{2}{3 v_{d}(k)} P_{a c}^{*}(k+1) \\
i_{q}^{*}(k)=-\frac{2}{3 v_{d}(k)} Q_{a c}^{*}(k)
\end{gathered}
$$

where, $v_{d}$ is the $d$-axis component of the grid voltage obtained from the PLL. Typically, $Q_{a c}^{*}(k)=0$, and hence also $i_{q}^{*}(k)=$ 0 , because under normal operating condition the power factor has to be as high as possible.

\section{B. Predictions}

For each of the four variables used in the cost function, the one-step-head predictions are computed to perform the minimization and retrieve the optimal states for the GSC switches and the optimal phase-shift angle to be applied to the DAB.

1) GSC predictions: The dynamics of the dc-link capacitor voltage is described by

$$
C_{d c} \frac{d v_{d c}}{d t}=i_{d c}
$$

where $C_{d c}$ is the dc-link capacitance. By applying the Euler approximation for a sampling period $T_{s}$ to (9), it is possible to calculate the one-step-ahead prediction of the dc-link voltage as

$$
\hat{v}_{d c}(k+1)=v_{d c}(k)+\frac{T_{s}}{C_{d c}} \hat{i}_{d c}(k+1)
$$

here, $\hat{i}_{d c}(k+1)$ is the dc-link current prediction at instant $k+1$ calculated as

$$
\begin{aligned}
\hat{i}_{d c}(k+1)= & -S_{a}(k) i_{a}(k) \\
& -S_{b}(k) i_{b}(k)-S_{c}(k) i_{c}(k)
\end{aligned}
$$

where $i_{a}(k), i_{b}(k), i_{c}(k)$ are the values of the grid currents measured at the $k$-th instant and $S_{a}(k), S_{b}(k), S_{c}(k)$ are the switching state of the three legs of the GSC. According to the approach proposed in [15], the predictions of the active and reactive powers are calculated as

$$
\begin{aligned}
\hat{P}_{a c}(k+1) & =\operatorname{Re}\left\{\mathbf{V}_{g}(k+1) \overline{\mathbf{I}}_{g}(k+1)\right\} \\
& \approx \operatorname{Re}\left\{\mathbf{V}_{g}(k) \overline{\mathbf{I}}_{g}(k+1)\right\} \\
\hat{Q}_{a c}(k+1) & =\operatorname{Im}\left\{\mathbf{V}_{g}(k+1) \overline{\mathbf{I}}_{g}(k+1)\right\} \\
& \approx \operatorname{Im}\left\{\mathbf{V}_{g}(k) \overline{\mathbf{I}}_{g}(k+1)\right\}
\end{aligned}
$$

where $\mathbf{V}_{g}(k+1)$ and $\overline{\mathbf{I}}_{g}(k+1)$ are the grid voltage and complex conjugate current vectors at the instant $k+1$. These vectors are defined as $\mathbf{V}_{g}=\frac{2}{3}\left(v_{a}+\boldsymbol{\alpha} v_{b}+\boldsymbol{\alpha}^{2} v_{c}\right)$ and $\mathbf{I}_{g}=$ $\frac{2}{3}\left(i_{a}+\boldsymbol{\alpha} i_{b}+\boldsymbol{\alpha}^{2} i_{c}\right)$ respectively. Since the grid frequency is much lower than the sampling frequency $(50 \mathrm{~Hz}$ and $40 \mathrm{kHz}$, respectively) it is possible to consider $\mathbf{V}_{g}(k+1) \approx \mathbf{V}_{g}(k)$.

Similarly to the references calculations, $\hat{i}_{d}(k+1)$ and $\hat{i}_{q}(k+1)$ are calculated as

$$
\begin{gathered}
\hat{i}_{d}(k+1)=\frac{2}{3 v_{d}(k)} \hat{P}_{a c}(k+1) \\
\hat{i}_{q}(k+1)=-\frac{2}{3 v_{d}(k)} \hat{Q}_{a c}(k+1) .
\end{gathered}
$$

At every iteration of the algorithm, equations (10) to (15) are evaluated for each of the 8 possible states of the GSC that are listed in Table I.

2) Battery Current Prediction: In this work, phase-shift modulation is employed for the DAB due to its simple implementation. The MDCS-MPC approach proposed in [18] is considered in order to discretize the phase shift $\varphi$, which is a continuous variable in nature. To do so, the parameter $\Delta$ is introduced, and it represents the achievable precision of the process, i.e. the finest possible interval between two consecutive values of $\varphi$ that the algorithm is able to apply. $\Delta$ depends on the ratio between the switching frequency of the devices, $f_{s}$, and the clock frequency, $f_{c l k}$, of the digital control platform on which the algorithm is implemented, as expressed

$$
\Delta=2 \pi \frac{f_{s}}{f_{c l k}} .
$$

The proposed algorithm considers 9 different values of $\varphi(k+1)$ for each sampling period, calculated as

$$
\varphi(k+1)=\{\varphi(k)+t \Delta\} \quad, \quad t=[-4,3, \ldots, 3,4]
$$

where $\varphi(k)$ is the optimal phase shift selected at the previous iteration.

By means of two nested loops, the couple of indices $(i, j)$ such that $S(i)$ and $\varphi(j)$ minimizes (1) is retrieved, where $1 \leq i \leq 8$ and $1 \leq j \leq 9$. So, the 9 values of $\varphi$ are evaluated for each one of the 8 switches configuration of the GSC, and the minimization is carried out on 72 cases.

Considering the DAB lossless model [21], the power transferred by the dc-dc stage can be calculated as in

$$
P_{b a t}=\frac{n v_{d c} v_{b a t} \varphi(\pi-|\varphi|)}{2 \pi^{2} f_{s} L_{d a b}}
$$

If the battery power is written as $P_{b a t}=i_{b a t} v_{b a t}$, it is possible to calculate the battery current prediction $\hat{i}_{b a t}(k+1)$ as

$$
\hat{i}_{\text {bat }}(k+1)=\frac{n v_{d c}(k) \varphi(k+1)(\pi-|\varphi(k+1)|)}{2 \pi^{2} f_{s} L_{d a b}}
$$

where $-\pi<\varphi<\pi$ and $n$ is the transformer turn ratio.

TABLE I: Switching states of the GSC

\begin{tabular}{|c|cccccccc|}
\hline States & $\mathbf{1}$ & $\mathbf{2}$ & $\mathbf{3}$ & $\mathbf{4}$ & $\mathbf{5}$ & $\mathbf{6}$ & $\mathbf{7}$ & $\mathbf{8}$ \\
\hline$S_{a}$ & 0 & 1 & 1 & 0 & 0 & 0 & 1 & 1 \\
\hline$S_{b}$ & 0 & 0 & 1 & 1 & 1 & 0 & 0 & 1 \\
\hline$S_{c}$ & 0 & 0 & 0 & 0 & 1 & 1 & 1 & 1 \\
\hline
\end{tabular}




\section{N parameter Design}

As previously stated, the $N$ parameter is needed to slow down the dynamics of the voltage regulation with respect to the active power control. However, while designing the MPC algorithm it has been noted through simulations that the value of $N$, together with $i_{b a t}^{*}$, may affect the steady-state error of the dc-link voltage. Indeed, for a given value of $i_{\text {bat }}^{*}$, a larger $N$ provides a slower dynamics for the dc-link voltage but, at the same time, it also leads to a higher steady-state error of $v_{d c}(k)$. Similarly, for a fixed value of $N$, a larger $i_{b a t}^{*}$ leads to a larger steady-state error. Since, in general, $i_{b a t}^{*}$ is fixed by the application, an effective way to compensate the dclink voltage steady-state error is to act on $N$ by adjusting this parameter at each cycle: in general, a suitably large $N$ is needed whenever a fast variation occurs in either of the references $v_{d c}^{*}$ and $i_{b a t}^{*}$. On the contrary, a smaller value of $N$ is required to avoid the steady-state error. Therefore, in order to avoid the limitations deriving from keeping $N$ constant, at each iteration the algorithm checks if the present values of both references $v_{d c}^{*}$ and $i_{b a t}^{*}$ are equal to those at the previous cycle and, if a variation is detected, a larger value of $N$ is set. During the transient, the value of $N$ is decreased at each control step by a certain amount $\Delta N$, up to a minimum value, which is set when the system reaches the steady state and the zero-tracking error is achievable. The parameter $N$ and its decremental rate $\Delta N$ strongly affect the dynamic response of the system. In particular, they affect the overshoots and the undershoots of the control variables during transients, and the settling time.

In this work, values of $N$ and $\Delta N$ have been selected by following a trial-and-error approach. The general idea is to choose sufficiently high values of $N$ so to guarantee fast dynamics for the dc-link voltage while limiting the overshoots of the controlled variables, and finally to adjust $\Delta N$ to regulate the time needed to reach steady-state.

\section{Simulation AND Results}

The effectiveness of the proposed MPC algorithm has been tested using MATLAB/Simulink and PLECS block library; in particular, the MPC algorithm has been implemented as a discrete-time Matlab function while the switching model of the system has been modelled in PLECS. A characterization of the steady-state and dynamics performance achieved by the MPC control algorithm is provided, and the results are compared with a PI control scheme. The THD achieved in the two cases is also discussed.

\section{A. MPC steady-state and dynamic performance}

Fig. 5 shows how the choice of the decremental rate $\Delta N$ affects the dynamics of the MPC algorithm when a positive step of $100 \mathrm{~V}$ and a negative step of $50 \mathrm{~V}$ are applied to the dc-link voltage reference. At every variation in the dc-link voltage reference $N$ is set to 300 , according to the strategy discussed in Section II-C to achieve zero steady-state error. The corresponding profiles of the $N$ parameter are shown in the bottom plot in Fig. 5.

Table II summarizes the values of rise time $T_{r}$, fall time $T_{f}$ and settling time $T_{\text {set }}$ characterizing the dc-link voltage step response in the two cases depicted in Fig. 5. It can be seen that the higher the $\Delta N$ the faster is the dynamics of the controller, in particular the transient times when $\Delta N=1.2$ (blue line) are considerably faster of the ones when $\Delta N=$
TABLE II: Dynamic performance of the MPC control for two different $\Delta N$ when $N_{\text {pos }}=300, N_{\text {neg }}=125$ and of the PI control when $f_{0, v}=20 \mathrm{~Hz}, f_{0, i}=400 \mathrm{~Hz}$.

\begin{tabular}{|c|c|c|c|c|}
\cline { 2 - 5 } \multicolumn{1}{c|}{} & \multicolumn{2}{c|}{ Positive step } & \multicolumn{2}{c|}{ Negative step } \\
\hline Control system & $T_{r}[\mathrm{~ms}]$ & $T_{\text {set }}[\mathrm{ms}]$ & $T_{f}[\mathrm{~ms}]$ & $T_{\text {set }}[\mathrm{ms}]$ \\
\hline MPC, $\Delta N=0.2$ & 14.85 & 24.65 & 8.92 & 28.86 \\
MPC, $\Delta N=1.2$ & 8.95 & 12.24 & 3.7 & 5.36 \\
PI & 10.01 & 45.89 & 8.2 & 42.71 \\
\hline
\end{tabular}

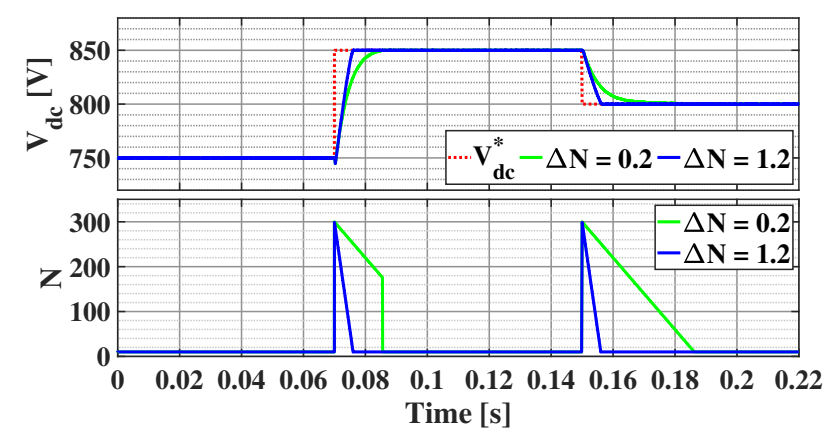

Fig. 5: dc-link voltage evolution.

0.2 (green line). Furthermore, when $\Delta N=0.2$ it can be seen that the algorithm avoids the overshoot by setting $N$ to $N_{\min }$ if the dc-link voltage reaches steady-state when $N$ is still larger than its minimum value.

\section{B. Comparison with conventional PI control}

The steady-state and dynamic performance have been tested by carrying out two simulations of $0.22 \mathrm{~s}$ each. In the first simulation $(\operatorname{Sim} 1)$ a positive step of $100 \mathrm{~V}$ and a negative step of $50 \mathrm{~V}$ are applied to the dc-link voltage reference (red line in Fig. 6 (b)) while keeping the $i_{b a t}^{*}$ constant at 70A. In the second simulation ( $\operatorname{Sim} 2$ ) a positive step of $30 \mathrm{~A}$ and a negative step of $20 \mathrm{~A}$ are applied to the battery current reference (dotted red line in Fig. 6 (a)) while the $V_{d c}^{*}$ was kept constant at $750 \mathrm{~V}$.

In FCS-MPC, the commutations of the converter's power switches do not occur at a fixed sampling frequency, leading to a spread harmonic spectrum, which affects power quality and makes the design of filters troublesome. In contrast to FCS-MPC, control techniques that include a modulation stage, such as VOC, have a fixed switching frequency and, therefore, their harmonic spectrum is well defined with harmonic packets centred on the carrier frequency and its multiples. In order to perform a meaningful comparison between these two approaches, the average switching frequency of the MPC working with a sampling time $T_{s}=25 \mu \mathrm{s}$ was estimated. The same value was set for the carrier in PWM modulator used in the a PI-based control algorithm which exploits a cascaded control configuration in which the external loop regulates the dc-link voltage, and the inner loop regulates the active and reactive power exchanged with the grid. The PI controllers have been tuned so that the voltage control loop has the largest achievable bandwidth, while still achieving an acceptable dynamic behaviour in terms of overshoot of the controlled variables. For the voltage control loop a bandwidth $f_{0, v}=20 \mathrm{~Hz}$ has been considered while a bandwidth $f_{0, i}=400 \mathrm{~Hz}$ has been chosen for the current control loop. A separate PI regulator is used in this scheme to control the battery current with the DAB using PSM. 


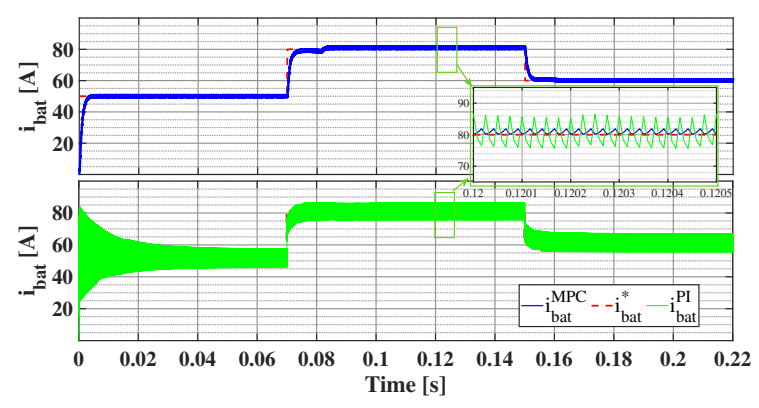

(a) Battery current $(\operatorname{sim} 1)$.

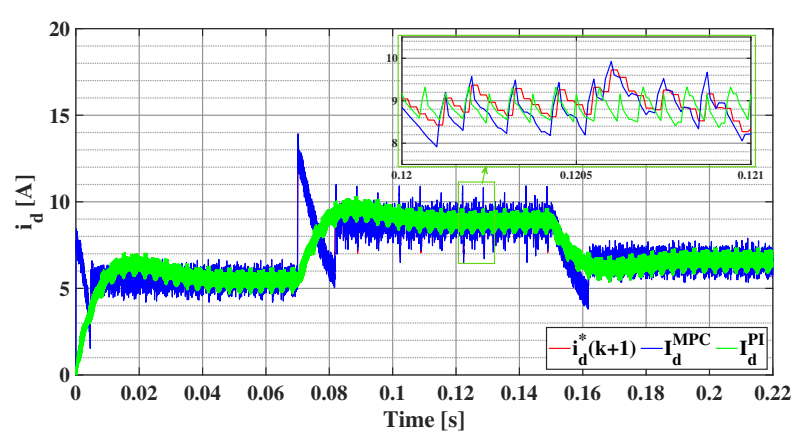

(c) $d$-axis current $(\operatorname{sim} 1)$.

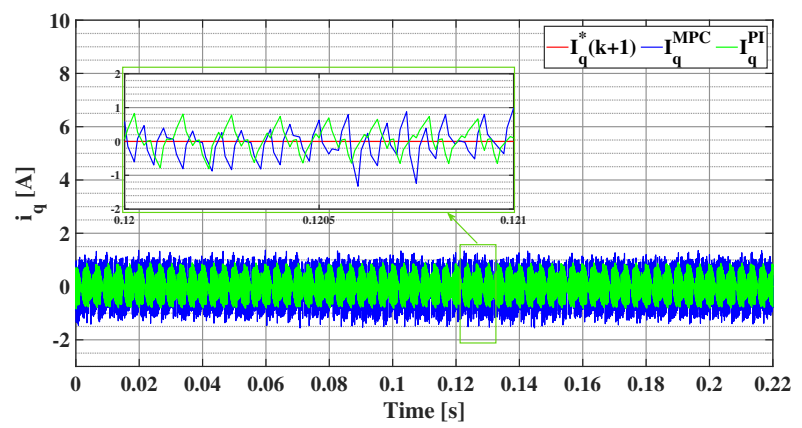

(e) $q$-axis current $(\operatorname{sim} 1)$.

Fig. 6: Simulation results, comparison of the proposed MPC $(\Delta N=1.2)$ with a conventional PI Controller $\left(f_{0 V}=20 H z\right.$, $\left.f_{0, i}=400 H z\right)$

The average switching frequency achieved with the proposed MPC has been obtained by computing the mean of the instantaneous switching frequency calculated as

$$
f_{s w}=\frac{\left\|S_{a b c}(k)-S_{a b c}(k-1)\right\|_{1}}{m T_{s}}
$$

where $\mathrm{m}$ is the number of switching devices, in this case, $m=6$. Considering a sampling frequency of $40 \mathrm{kHz}$, the resulting average switching frequency in the MPC scheme is $8 \mathrm{kHz}$.

Figs. 6 shows the results of the comparison for the two simulations. In particular siml is shown in Fig. 6(b), while sim2 in Figs. 6(a) 6(c), 6(d), 6(e), and 6(f).

Fig. 6 (a) shows the evolution of the battery current. The feedback tracks both steps with fast dynamics. The rise time detected during the reference positive step is in the order of $2.3 \mathrm{~ms}$; during the negative step, the measured fall time is approximately $1.7 \mathrm{~ms}$. Both controls have no steady-state error and a comparable dynamics behaviour. However the MPC has a remarkably lower current ripple at steady state.

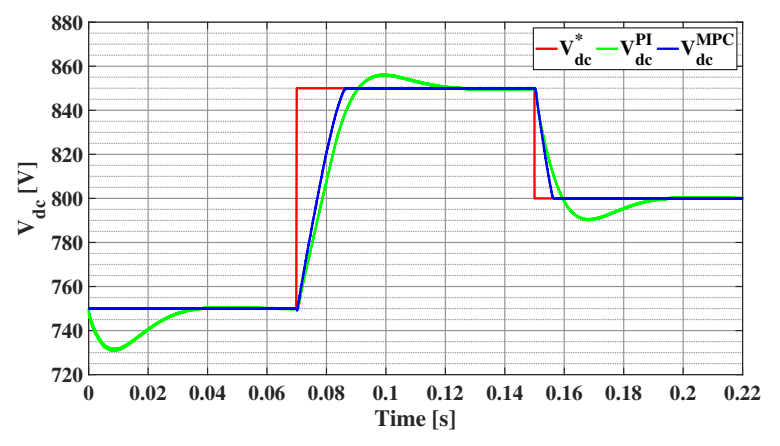

(b) dc-link voltage $(\operatorname{sim} 2)$.

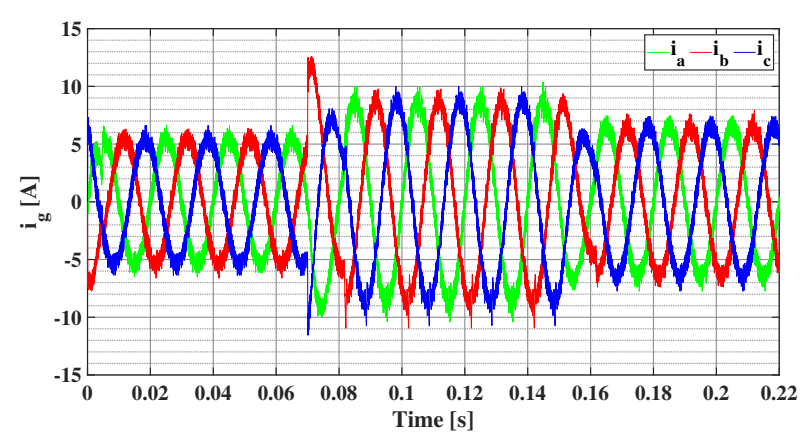

(d) Grid current MPC ( $\operatorname{sim} 1)$.

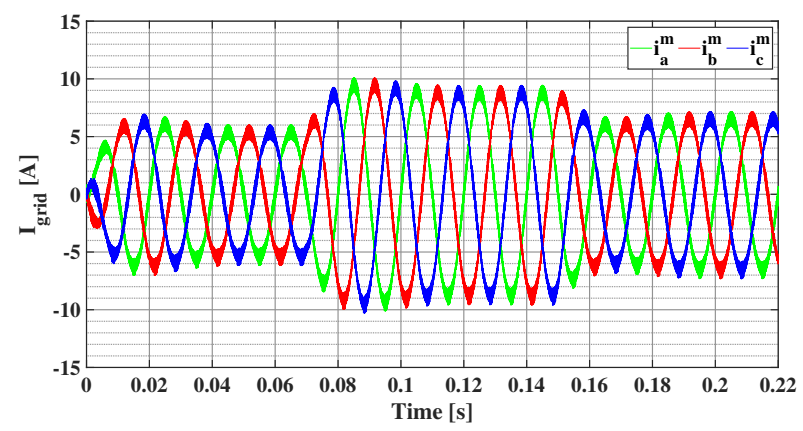

(f) Grid current PI $(\operatorname{sim} 1)$.

Fig. 6 (b) compares the dc-link voltage step response of the MPC algorithm with the described PI control system. The corresponding dynamics performance are specified in the second and third rows of Table II, respectively. As it can be observed, the two responses have comparable rise/fall times but in the PI response the settling time is much larger and an overshoot of $9.2 \mathrm{~V}$ and an undershoot of $7.3 \mathrm{~V}$ can be observed.

Fig. 6 (c) shows the comparison of the MPC with the PI when tracking the one-step-ahead reference of $d$-axis current. The MPC during both positive and negative steps has a faster dynamics than the PI, even though it presents a greater overshoot (up to $14 \mathrm{~A}$ during the positive step) and undershoots. Fig. 6 (e) shows the comparison of the MPC with the PI when tracking the reference of $q$-axis current. In this case the MPC and the PI have similar behaviour.

Figs. 6(d) and 6(f) show the grid current wave forms; the former refers to the FCS-MPC, the latter to the PIbased control. Table III summarizes the THD of the grid current achieved when three different values of battery current reference are considered in the two cases: when the two 
models work with the same switching frequency the resulting THD are comparable even if the scheme including the PWM modulator achieves slightly better performance.

TABLE III: THD measured for the phase $a$ of $i_{\text {grid }}$ considering 3 different values of $i_{b a t}$.

\begin{tabular}{|c|c|c|}
\hline$i_{\text {bat }}$ & $T H D_{M P C}[\%]$ & $T H D_{P I}[\%]$ \\
\hline $50 \mathrm{~A}$ & 11.1 & 8.5 \\
\hline $80 \mathrm{~A}$ & 7.5 & 5.2 \\
\hline $60 \mathrm{~A}$ & 9.2 & 7.1 \\
\hline
\end{tabular}

\section{CONCLUSION AND Future Works}

In this paper, a model predictive algorithm has been proposed for the control of a double-stage ac-dc conversion system interfacing a vanadium redox flow battery with the power grid. The converter considered for the application is composed by a three-phase inverter/rectifier on the grid side in cascade with a dual active bridge. The novelty of the proposed approach is the unification of the control actions of both conversion stages into a single optimization function, achieving controlled power transfer between the grid and the battery and regulation of grid-side electrical quantities with fast dynamics and flexible implementation. The proposed predictive control has been tested in simulation and compared to a conventional PI-based control system, showing a better dynamic performance while achieving a comparable quality of the voltage and current waveforms. Switching from a finitecontrol-set approach to a modulated predictive control may result in better power quality, especially on the grid side.

Future research work will be addressed to the experimental implementation of the proposed model predictive control system in order to evaluate its effective computational burden and performance with respect to conventional approaches. Further interest is directed to including advanced features within the cost function to fully exploit the potentiality of the proposed algorithm, such as including the battery state of charge estimation to improve system reliability and efficiency or adopting more flexible modulation approaches to reduce converter losses.

\section{REFERENCES}

[1] R. Lasseter, "Microgrids," in 2002 IEEE Power Engineering Society Winter Meeting. Conference Proceedings (Cat. No.02CH37309), vol. 1, 2002, 305-308 vol.1. DOI: 10.1109/ PESW.2002.985003.

[2] G. Pepermans, J. Driesen, D. Haeseldonckx, R. Belmans, and W. D'haeseleer, "Distributed generation: Definition, benefits and issues," Energy Policy, vol. 33, no. 6, pp. 787-798, 2005, ISSN: 0301-4215. DOI: https://doi.org/10.1016/j.enpol.2003. 10.004. [Online]. Available: https://www.sciencedirect.com/ science/article/pii/S0301421503003069.

[3] A. Bhattacharjee and H. Saha, "Design and experimental validation of a generalised electrical equivalent model of vanadium redox flow battery for interfacing with renewable energy sources," Journal of Energy Storage, vol. 13, pp. 220232, 2017.

[4] M. Guarnieri, P. Mattavelli, G. Petrone, and G. Spagnuolo, "Vanadium redox flow batteries: Potentials and challenges of an emerging storage technology," IEEE Industrial Electronics Magazine, vol. 10, no. 4, pp. 20-31, 2016.

[5] S. Inoue and H. Akagi, "A bidirectional dc-dc converter for an energy storage system with galvanic isolation," IEEE Transactions on Power Electronics, vol. 22, no. 6, pp. 22992306, 2007. DOI: 10.1109/TPEL.2007.909248.
[6] D. Christen and J. Biela, "Analytical switching loss modeling based on datasheet parameters for mosfets in a half-bridge," IEEE Transactions on Power Electronics, vol. 34, no. 4, pp. 3700-3710, 2019. DOI: 10.1109/TPEL.2018.2851068.

[7] J. Rocabert, A. Luna, F. Blaabjerg, and P. Rodríguez, "Control of power converters in ac microgrids," IEEE Transactions on Power Electronics, vol. 27, no. 11, pp. 4734-4749, 2012. DOI: 10.1109/TPEL.2012.2199334.

[8] H. Qin and J. W. Kimball, "Closed-loop control of dc-dc dual-active-bridge converters driving single-phase inverters," IEEE Transactions on Power Electronics, vol. 29, no. 2, pp. 1006-1017, 2014. DOI: 10.1109/TPEL.2013.2257859.

[9] A. M. Bouzid, J. M. Guerrero, A. Cheriti, M. Bouhamida, P. Sicard, and M. Benghanem, "A survey on control of electric power distributed generation systems for microgrid applications," Renewable and Sustainable Energy Reviews, vol. 44, pp. 751-766, 2015.

[10] P. Karamanakos and T. Geyer, "Guidelines for the design of finite control set model predictive controllers," IEEE Transactions on Power Electronics, vol. 35, no. 7, pp. 7434-7450, 2019.

[11] P. Karamanakos, E. Liegmann, T. Geyer, and R. Kennel, "Model predictive control of power electronic systems: Methods, results, and challenges," IEEE Open Journal of Industry Applications, vol. 1, pp. 95-114, 2020.

[12] S. Vazquez, J. I. Leon, L. G. Franquelo, J. Rodriguez, H. A. Young, A. Marquez, and P. Zanchetta, "Model predictive control: A review of its applications in power electronics," IEEE industrial electronics magazine, vol. 8, no. 1, pp. 1631, 2014

[13] S. Vazquez, J. Rodriguez, M. Rivera, L. G. Franquelo, and M. Norambuena, "Model predictive control for power converters and drives: Advances and trends," IEEE Transactions on Industrial Electronics, vol. 64, no. 2, pp. 935-947, 2016.

[14] J. Rodriguez and P. Cortes, "Predictive control of permanent magnet synchronous motors," in Predictive Control of Power Converters and Electrical Drives. 2012, pp. 133-144. DOI: 10.1002/9781119941446.ch9.

[15] _ - "Control of an active front-end rectifier," in Predictive Control of Power Converters and Electrical Drives. 2012, pp. 81-98. DOI: 10.1002/9781119941446.ch6.

[16] - "Predictive control of a three-phase inverter," in Predictive Control of Power Converters and Electrical Drives. 2012, pp. 41-63. DOI: 10.1002/9781119941446.ch4.

[17] J. Rodriguez, M. P. Kazmierkowski, J. R. Espinoza, P. Zanchetta, H. Abu-Rub, H. A. Young, and C. A. Rojas, "State of the art of finite control set model predictive control in power electronics," IEEE Transactions on Industrial Informatics, vol. 9, no. 2, pp. 1003-1016, 2013. DOI: 10.1109/ TII.2012.2221469.

[18] L. Chen, S. Shao, Q. Xiao, L. Tarisciotti, P. W. Wheeler, and T. Dragičević, "Model predictive control for dual-activebridge converters supplying pulsed power loads in naval dc micro-grids," IEEE Transactions on Power Electronics, vol. 35, no. 2, pp. 1957-1966, 2019.

[19] L. Chen, F. Gao, K. Shen, Z. Wang, L. Tarisciotti, P. Wheeler, and T. Dragičević, "Predictive control based dc microgrid stabilization with the dual active bridge converter," IEEE Transactions on Industrial Electronics, vol. 67, no. 10, pp. 8944-8956, 2020.

[20] P. Zanchetta, P. Cortes, M. Perez, J. Rodriguez, and C. Silva, "Finite states model predictive control for shunt active filters," in IECON 2011-37th Annual Conference of the IEEE Industrial Electronics Society, IEEE, 2011, pp. 581-586.

[21] F. Krismer and J. W. Kolar, "Accurate small-signal model for the digital control of an automotive bidirectional dual active bridge," IEEE transactions on power electronics, vol. 24, no. 12, pp. 2756-2768, 2009. 